\title{
Clinical use of antibacterial mesh envelopes in cardiovascular electronic device implantations
}

This article was published in the following Dove Press journal:

Medical Devices: Evidence and Research

12 January 2015

Number of times this article has been viewed

\author{
David S Hirsh ${ }^{1,2}$ \\ Heather L Bloom ${ }^{1,2}$ \\ 'Department of Cardiovascular \\ Medicine, School of Medicine, \\ Emory University, Atlanta, GA, USA; \\ ${ }^{2}$ Department of Cardiovascular \\ Medicine, Atlanta Veterans Affairs \\ Medical Center, Decatur, GA, USA
}

Correspondence: Heather L Bloom Department of Cardiovascular Medicine, Atlanta Veterans Affairs Medical Center, 1670 Clarimont Road, Decatur,

GA 30033, USA

Tel + I 9165483563

Email hbloom@emory.edu

\begin{abstract}
Cardiovascular implantable electronic device system infection is a serious complication of cardiac device implantation and carries with it a risk of significant morbidity and mortality. In the last 15 years, expansions of indications for cardiac devices have resulted in much higher volumes of much sicker patients being implanted, carrying significant risk of infection. Coagulase (-) Staphylococcus and Staphylococcus aureus are responsible for the majority of these infections, and these organisms are increasingly resistant to methicillin. The Aigis ${ }^{\mathrm{TM}}$ envelop is a Food and Drug Administration-approved implantable mesh that is impregnated with antibiotics that can be placed in the surgical incision prior to closure. The antibiotics elute off the mesh for 7-10 days, providing in vivo surgical site coverage with rifampin and minocyclin. This paper reviews the three retrospective clinical trials published in peer-reviewed journals and the interim analysis of the two ongoing prospective trials that have been presented at international conferences. Overall consensus is that the Aigis ${ }^{\mathrm{TM}}$ offers significant risk reduction for cardiovascular implantable electronic device infection. We then give a comprehensive discussion of how to use the Aigis ${ }^{\mathrm{TM}}$ envelop in the clinical setting, comparing the manufacturer's recommendations with our extensive clinical experience.
\end{abstract}

Keywords: pacemaker, defibrillator, cardiac electronic implantable device, infection, Aigis ${ }^{\mathrm{TM}}$

Cardiovascular implantable electronic device (CIED) system infection is a serious complication of cardiac device implantation and is associated with up to an $18 \%$ mortality rate. Studies vary on the incidence of CIED system infection, but the most contemporary data set addressing this issue was a query of nationwide discharge records. This study revealed a startling increase in CIED system infections. From 1993 to 2008, the overall incidence of CIED system infection during the study period was noted to be $1.61 \%$. However, co-incident with the rise in implantable cardioverter-defibrillator (ICD) implantation, which represented $35 \%$ of all implants by the end of the study period, there was a $210 \%$ increase in CIED system infections with an incidence rate of $2.41 \%$ by $2008 .{ }^{1}$ Untreated device-related infection is associated with extremely high morbidity and mortality, with mortality rates reported as high as $66 \%{ }^{2}$

\section{Treatment}

CIED system infections generally require extraction of the entire system along with a prolonged course of antibiotics. ${ }^{3}$ It is worth mentioning that the procedure to remove an infected system via laser lead extraction may carry up to a $0.8 \%$ in-hospital mortality rate. ${ }^{4}$ Moreover, following successful system extraction, temporary strategies often 
need to be employed to treat and/or protect the patient as they recover from their infection. For example, in the case of pacemaker-dependent patients, an adequate and safe temporary pacing strategy needs to be employed prior to the implantation of a new permanent system or a Life Vest in the case of those at high risk for sudden cardiac death. These bridging treatment modalities have associated risks and costs.

\section{Economic impact}

From an economic perspective, CIED system infection represents a tremendous burden. By 2008, in-hospital charges for CIED system were in the range of $\$ 146,000 .{ }^{5}$ Effective in October 2012, CMS (Center for Medicare and Medicaid Services) in the USA added CIED infections to their list of hospital-acquired infections for which they will not reimburse. Thus, from both a medical and economic perspective, all measures that can optimally reduce CIED system infections ought to be employed at the time of implant and/or generator exchange.

\section{Risk factors}

The risk factors for CIED system infection have been well described in the literature and include diabetes mellitus, congestive heart failure, renal failure, generator exchange, preoperative fever, peri-procedural temporary pacing, early reintervention, number of leads implanted, use of oral anticoagulants, and use of corticosteroids, in addition to advanced age. Implantation of a new system and peri-procedural antibiotics have both been associated with reduced risks of CIED system infection. ${ }^{3}$

\section{Microbiology}

Coagulase (-) Staphylococcus (eg, Staphylococcus epidermidis) and Staphylococcus aureus are responsible for about $70 \%$ of CIED infections, ${ }^{1-5}$ and nosocomial isolates of these pathogens are becoming increasingly resistant to methicillin (equivalent to cefazolin resistance). ${ }^{6,7} \mathrm{~A}$ recent study from the Mayo Clinic in 2008 observed that $41 \%$ of CIED infections were due to Coagulase (-) Staphylococcus and $41 \%$ due to $S$. aureus, with gram-negative rods, fungi, and mycobacterium contributing small numbers. ${ }^{8}$

A characteristic of many pathogenic strains of $S$. epidermidis and $S$. aureus is the production of a biofilm. Secreted teichoic acid, normally found in the cell walls, aids in the formation of the biofilm. Biofilms occur when populations of free-floating microorganisms become attached to each other and form an adhesive bond that develops into a dense matrix. This ability to form a biofilm on the surface of a prosthetic device is a significant determinant of virulence for this bacterium. In vitro work has demonstrated that Staphylococcus have a strong ability to adhere to plastic and metal surfaces. The biofilm matrix grows through the addition of more microorganisms and through cell division of microorganisms that are already a part of this matrix. Secretion of cellular signals results in recruitment of specific types of bacterium, which protect the biofilm colonies. The biofilm matrix is physically dense, with the outer layers of microorganisms protecting the inner colony. This protective behavior means those biofilm bacteria are up to one thousand times more resistant to antimicrobial agents than free-floating organisms. ${ }^{9}$ The biodegradative effects of $S$. aureus and macrophages on silicone insulation have been studied in explanted leads. Kolodzinska et al reviewed leads removed from eight patients for both infective and noninfective indications. Leads were examined, then fragmented, cultured with $S$. aureus strains, and then re-examined with scanning microscopes. All strains demonstrated biodegradation of silicone insulation. ${ }^{10}$

In addition, the reaction of the human body to foreign bodies is a complex process. It is well known in the surgical literature that different synthetic and biological scaffoldings alter the healing and inflammatory process. Especially in the subacute surgical phase (1-4 weeks), biomaterial/body interface properties play an important role in modulating protein absorption, monocycte/macrophage adhesion, and alter tissue remodeling. At this point, we do not have specific data on the interactions between the Aigis ${ }^{\mathrm{TM}}$ and the human body. These characteristics are the reason that guidelines mandate complete hardware removal for Staphylococcus and Streptococcus bacteremia in the presence of CIED hardware.

\section{What preventative treatment options are available?}

Standard sterile surgical precautions and preparation are a mainstay of device implantation. Contamination during the surgical procedure itself has long been well known to be the major source of surgical infections. Guidelines recommend preoperative intravenous antibiotics. Data, however, are not definitive about whether postoperative oral antibiotics are useful, and tissue perfusion is poor due to the lack of vascularity in a pacemaker capsule. Thus, the ability to implant antibiotics directly in the surgical incision itself is a logical step.

The only internal implantable source of antibiotics that is currently Food and Drug Administration (FDA) approved is the Aigis ${ }^{\mathrm{TM}}$ implantable prosthesis. The Aigis ${ }^{\mathrm{TM}}$ is an implantable mesh that is impregnated with antibiotics that can be placed in the surgical incision prior to closure. The 
antibiotics elute off the mesh for 7-10 days, giving a strong concentration of prophylaxis in the tissue, a vast improvement over poor bioavailability of oral agents in tissue.

The Aigis ${ }^{\mathrm{TM}}$ comes in two types, $100 \%$ absorbable and nonabsorbable. Both Aigis ${ }^{\mathrm{TM}}$ are dual-component (resorbable and nonresorbable), sterile prosthesis constructed of openpore weave, knitted filaments of a lightweight mesh and coated with a resorbable polymer impregnated with antimicrobial agents: minocycline and rifampin. Minocycline acts as a bacteriostatic to inhibit protein synthesis while rifampin acts as a bacteriocidal that inhibits DNA-dependent RNA polymerase. Both agents are impregnated into the resorbable polymer in concentrations of $86 \mu \mathrm{g} / \mathrm{cm}^{2}$. Minocycline has an antimicrobial spectrum against a wide range of gram-positive and gram-negative organisms. Rifampin is a semisynthetic compound derived from Amycolatopsis rifamcinica. Rifampin has antimicrobial activity against select gram-positive and gram-negative organisms. The mesh is the part that varies between the two types, consisting of either polypropylene- or a tyrosine-based resorbable polymer. Polypropylene has been utilized as a medical device implant for surgery for decades and has undergone extensive testing and observations. The most common use is hernia repairs. The absorbable tyrosinebased polymer is designed to degrade to natural metabolites with a known history. It has been demonstrated to resorb benignly, in the same manner as resorbable surgical sutures, while eliciting a minimal inflammatory response. It also has a long history of use with other FDA-approved implantable medical devices.

\section{Summary of published data on the Aigis ${ }^{\mathrm{TM}}$ to date Animal data}

The Aigis ${ }^{\mathrm{TM}}$ envelop was FDA approved on June 16, 2008, and since that time over 25,000 implants have been performed in the USA. The anxiously awaited absorbable version was first released in January 2013 in Canada, finally following in the USA in July 2013. Initial FDA approval was based on the trials performed in animal models. The first trial was performed in rabbits, which received pacing devices with or without the Aigis ${ }^{\text {TM }}$ envelope via a standard surgical approach. After closure, a dose of bacteria was injected directly into the closed pocket. Organisms included S. aureus, S. epidermidis (S. Epi), Staphylococcus capitis, and Escherichia coli, $105 \mathrm{CFU}$ (colony-forming units). The animals were sacrificed and devices extracted 7 days later. Assessment for bacterial presence was performed with imaging scanning electron microscopy and laser scanning confocal microscopy. All the devices treated with the Aigis ${ }^{\mathrm{TM}}$ patches were sterile. ${ }^{11,12} \mathrm{~A}$ similar model was also performed in canines, with methicillin-resistant S. aureus. Again, 100\% efficacy was observed in the prevention of bacterial contamination and infection (TyRx, personal communication, June, 2013).

\section{Retrospective human data: three trials}

The first human trial, known as the Cooperative Multicenter study Monitoring a CIED Antimicrobial Device (COMMAND) study was a multicenter retrospective cohort trial (Tables 1 and 2). It enrolled 624 individuals who were undergoing either initial implant or replacement/ revisions for pacemakers, ICDs, or cardiac resynchronization devices (CRT-D and CRT-P) and received the Aigis ${ }^{\text {TM }}$ envelop based on the clinical decision of the implanting physicians. Sites included academic, community, and a

Table I Patient characteristics: retrospective studies

\begin{tabular}{|c|c|c|c|}
\hline Trials & COMMAND & Vanderbilt & $\begin{array}{l}\text { Arrhythmia } \\
\text { institute }\end{array}$ \\
\hline \multicolumn{4}{|l|}{ Aigis ${ }^{\mathrm{TM}}$ present } \\
\hline \multicolumn{4}{|l|}{ Aigis $^{\mathrm{TM}}$} \\
\hline $\begin{array}{l}\% \text { decrease in infection } \\
\text { with Aigis }{ }^{\mathrm{TM}}\end{array}$ & NA & $95 \%(P=0.04)$ & $70 \%(P=0.048)$ \\
\hline Number of patients & 624 & 929 & 1,240 \\
\hline Follow-up & 1.9 months & 18 months & 6 months \\
\hline \multicolumn{4}{|l|}{ Device type } \\
\hline PPM & 35 & NR & $61 \%$ \\
\hline ICD & 65 & NR & $22 \%$ \\
\hline CRT & 40 & NR & $17 \%$ \\
\hline \multicolumn{4}{|l|}{ Procedure type } \\
\hline Initial & $32 \%$ & $63 \%$ & $66 \%$ \\
\hline Replacement & $68 \%$ & $37 \%$ & $34 \%$ \\
\hline \multicolumn{4}{|c|}{ Patient characteristics/risk factors } \\
\hline Age & $70 \pm 13$ & $67 \pm 11$ & $77 \pm 11$ \\
\hline Sex, \% male & $68 \%$ & $63 \%$ & $62 \%$ \\
\hline Diabetes & $39 \%$ & $48.6 \%$ & $31 \%$ \\
\hline $\mathrm{CHF}$ & $60 \%$ & NR & $34 \%$ \\
\hline Renal disease & $35 \%$ & $42 \%$ & NR \\
\hline Oral AC & $35 \%$ & $63 \%$ & $33 \%$ \\
\hline Corticosteroids & $7 \%$ & $12 \%$ & $5 \%$ \\
\hline Temp pacing & $2 \%$ & NR & $N R$ \\
\hline Number of leads & $42 \%, 2+$ & $37 \%, 3+$ & $N R$ \\
\hline Early redo & $2 \%$ & $6 \%$ & $N R$ \\
\hline PPM dependent & NR & $30 \%$ & NR \\
\hline Fever/elevated WBC & NR & $24 \%$ & NR \\
\hline Prior CEID infection & NR & $5 \%$ & NR \\
\hline
\end{tabular}

Abbreviations: AC, anticoagulation; CEID, cardiac electronic implantable device; $\mathrm{CHF}$, congestive heart failure; COMMAND, Cooperative Multicenter study Monitoring a CIED Antimicrobial Device; CRT, cardiac resynchronization therapy; ICD, internal cardioverter-defibrillator; NA, not applicable; NR, not recorded; PPM, pacemaker; Temp, temporary. 
Table 2 Infection rates: prospective trials

\begin{tabular}{lll}
\hline Trial & \multicolumn{2}{l}{ Citadel/Centurion } \\
\hline $\begin{array}{l}\text { Number of patients } \\
\text { Follow-up }\end{array}$ & 1,100 & \\
$\begin{array}{l}\text { Device type } \\
\text { PPM }\end{array}$ & 3 months & 6 months \\
ICD & $0 \%$ & $0 \%$ \\
CRT & $40 \%$ & $40 \%$ \\
Procedure type & $60 \%$ & $60 \%$ \\
$\quad$ Initial & $0 \%$ & \\
$\quad$ Replacement & $100 \%$ & $0 \%$ \\
Major infections & $0.1 \%(I)$ & $0.2 \%(2)$ \\
\% decrease in infections & $95 \%$ & $89 \%$ \\
compared to historic controls & & \\
\hline
\end{tabular}

Abbreviations: CRT, cardiac resynchronization therapy; ICD, internal cardioverterdefibrillator; PPM, pacemaker.

Veterans Administration hospital. Patients had a mean age of $70,32 \%$ were women, and $22 \%$ were Black or Hispanic. From a literature review, nine high-risk factors were selected that could be obtained from hospital records and were associated with increased risk of infection, with odds ratios ranging from 2.46 (temporary pacing wires) to 15.04 (early reintervention). Patients had an average of $2.5( \pm 1.4)$ risk factors of the nine predefined for infection. They were followed up for a mean of 2 months (1.9 \pm 2.4$)$ and three infections were observed (at 9, 11, and 146 days), all three in patients with replacements and/or revision procedures, for an overall rate of $0.48 \%$. Both blood and wound cultures were negative in the first two patients. The third patient had a chronic nonhealing incision that was followed up for 146 days until the device became exposed and grew out coagulase-negative $S$. aureus from the wound site and generator. This subdivides to represent a $0 \%$ infection rate for new implants and a $0.72 \%$ rate for replacements, significantly lower than historically published controls. ${ }^{13}$

A retrospective, single-site trial was performed at Vanderbilt Heart and Vascular Institute. They examined 260 adult patients who underwent CIED placement between November 2009 and April 2012 and received the Aigis ${ }^{\text {TM }}$ envelop. Risk factors utilized as criteria to decide to implant the Aigis ${ }^{\mathrm{TM}}$ were prospectively selected, and patients needed to have two or more of the following risk factors: diabetes, renal insufficiency (creatinine $\geq 1.5 \mathrm{mg} / \mathrm{dL}$ ), systemic anticoagulation, chronic daily steroid use, prior documented CIED infection, three or more transvenous leads, pacemaker dependence, early pocket reentry (defined as within 2 weeks) or fever of $100.5^{\circ} \mathrm{F}$ or higher, or white blood cell count of 11,000 or higher within 24 hours of the procedure. These patients were matched to a control population of 639 adult patients who also had two or more risk factors and had underwent device implantation in the 18 months prior to the use of the Aigis ${ }^{\mathrm{TM}}$ at that institution. Mean patient age was 66 years, $70 \%$ were male, and the mean number of risk factors was $2.8 \pm 1.2$. After a mean of $18 \pm 7$ months of follow-up (with a minimum of 90 days), there was $1(0.4 \%)$ major infection in the Aigis ${ }^{\mathrm{TM}}$ group versus 19 (3.0\%) in the control group. The single infection occurred in a 77-yearold male with congestive heart failure, atrial fibrillations, chronic anticoagulation, and a CRT-D device that was moved from a left anterior chest site to the right to facilitate local radiation for lung cancer. The right-sided device had poor wound healing, and it was re-relocated back to the left side. Two months after the second relocation, he had skin erosion and underwent pocket revision, and in this final procedure, the Aigis ${ }^{\mathrm{TM}}$ envelope was utilized. He developed a surgical site infection with blood cultures positive for Pseudomonas aeruginosa. All hardware was removed. ${ }^{14}$

A second single-site, retrospective, dual-cohort trial from the Arrhythmia Institute in Ridgewood New Jersey was recently published in Heart Rhythm April 2014. Mittal et al examined their patient data in the 2 years prior to the utilization of the Aigis ${ }^{\mathrm{TM}}$ envelop and compared it to the following 2 years after implementation. They identified 1,651 consecutive patients between January 2007 and October 2009, prior to Aigis ${ }^{\mathrm{TM}}$, and 1,240 between October 2009 and September 2011. Of the 1,240, 275 patients received the Aigis ${ }^{\mathrm{TM}}$ envelope based on physician preference. The features associated with the decision to utilize Aigis ${ }^{\mathrm{TM}}$ were male sex, congestive heart failure, diabetes mellitus, CRT or ICD, and replacement/revision. Follow-up data were examined at 6 months. Overall, 33 (1.1\%) patients developed major infection requiring hardware extraction. Eleven (3.8\%) developed bacteremia due to Staph and Strep organisms. Twenty-five $(8.6 \%)$ had pocket infections without bacteremia. Patient characteristics were similar to previous trials. Risk factor logistic regression analysis identified seven independent risk factors, consistent with previous trials. However, here the authors developed a composite point score from 0 to 25 with risk factors assigned a point score based on their LR weight. Patients were divided into three groups by point score and compared between the "pre-" and "post-" Aigis eras. In the pre-Aigis ${ }^{\mathrm{TM}}$ era, infection rates were $1.0 \%$ in the lowest tertile, $3.4 \%$ in the middle tertile, and $11.1 \%$ in the highest tertile. In the post-Aigis ${ }^{\mathrm{TM}}$ cohort, infection rates between tertiles showed no difference, at $1.4 \%, 0.7 \%$, and $0.0 \%$, respectively. Of the 456 patients in the post analysis, $34 \%$ of the low-risk and $88 \%$ of highest risk tertiles received an Aigis ${ }^{\mathrm{TM}}$ envelop. To reiterate, when $88 \%$ of the highest 
risk patients received the Aigis ${ }^{\mathrm{TM}}$, the overall 6-month infection rate was 0 , compared to the 6 -month infection rate of $11 \%$ at the same center in the preceding 2 years when Aigis $^{\mathrm{TM}}$ was unavailable. ${ }^{15}$

\section{Prospective human data: Citadel (Clinical trials: NCTO I04386 I) Centurion (CT NCT0I043705)}

Two prospective trials have completed enrolment and have presented interim results with the Aigis ${ }^{\mathrm{TM}}$ envelope, the Centurion and Citadel trials (Table 3). Both enrolled patients undergoing generator change outs for all types of CIEDs. In the Citadel study, it was planned that patients receive Aigis ${ }^{\mathrm{TM}}$ envelope with ICD generator change out and be compared to published controls who have undergone device generator change outs. Centurion was designed to enroll patients undergoing CRT device change out, to be compared to casematched controls. Follow-up is planned for 12 months, with interim analysis presented for 3 and 6 months.

The 90-day (3-month) interim analysis was presented at the Heart Rhythm Society annual scientific sessions in May 2013. The first 1,000 patients who completed 3-month follow-up were analyzed. Data were obtained for 55 centers. Mean patient age was $71,24 \%$ were women and $14 \%$ were non-Caucasian. The combined cohort had a $0.1 \%$ rate of major infection $(\mathrm{n}=1)$ and a $1.1 \%$ rate of superficial skin cellulitis $(n=11)$. This represents $94 \%-95 \%$ fewer major infections than the predefined published control cohorts: at 81 days Gould and $\mathrm{Krahn}^{16}$ reported $1.88 \%(P=0.001)$ and the Ontario ICD database ${ }^{17}$ reported a 45 -day major infection rate of $1.7 \%(P=0.001)$. No unanticipated serious events were reported. ${ }^{18}$ The 6-month follow-up data were presented in June 2014 at Cardiostim in Nice, France. Overall major infection rate was $0.2 \%(\mathrm{n}=2)$ as compared to $1.88 \%$ and $1.67 \%$ (see prior) for a relative reduction of $88 \%-89 \%{ }^{19}$ Citadel is powered to detect a $1.48 \%$ absolute decrease in 12 -month infection rates. Centurion is powered to detect a $1.11 \%$ absolute decrease in infection rates.

Table 3 Summary of prospective trials with Aigis ${ }^{\mathrm{TM}}$ : Citadel and Centurion

\begin{tabular}{lllll}
\hline $\begin{array}{l}\text { Follow-up for } \\
\text { Citadel and } \\
\text { Centurion }\end{array}$ & $\begin{array}{l}\text { Major } \\
\text { infections } \\
\%, \mathbf{n}\end{array}$ & $\begin{array}{l}\text { Historic } \\
\text { controls (Gould } \\
\text { and Krahn) }\end{array}$ & $\begin{array}{l}\text { \% decrease } \\
\text { in infection }\end{array}$ & $\boldsymbol{P}$ \\
\hline 90 days & $0.1 \%(\mathrm{I})$ & $1.88 \%$ & $95 \%$ & $<0.001$ \\
& & $1.67 \%$ & $94 \%$ & $<0.001$ \\
180 days & $0.2 \%(2)$ & $1.88 \%$ & $89 \%$ & $<0.001$ \\
& & $1.67 \%$ & $88 \%$ & $<0.01$ \\
\hline
\end{tabular}

The Aigis ${ }^{\mathrm{TM}}$ is FDA approved for other surgical procedures, in addition to cardiac procedures. In July 2013, the Aigis $^{\mathrm{TM}}$ envelope received FDA approval for use in neurosurgical hardware for vagal neural stimulators. A few months later, use in spinal modulator hardware was also approved.

\section{How to use the Aigis ${ }^{\mathrm{TM}}$ : company guidelines and personal experience}

The Aigis ${ }^{\mathrm{TM}}$ pouch is implanted at point in the surgery when the leads have been implanted and the suture sleeves have been secured to the underlying tissue, consistent with your typical practice. The pulse generator should be attached to the leads in the standard fashion. We recommend that you insert the device in the pocket prior to utilizing the pouch and test two things. First, do your standard interrogation of $\mathrm{R}$ and/or $\mathrm{P}$ waves, impedances and thresholds to be sure that your leads are stable in the heart and the set screws are well deployed. Then observe that you have sufficient space in the pocket to accommodate the additional volume of the pouch and still have good tissue approximation of your incision. Recalling that surgical sites closed under too much tension are higher risk of wound dehiscence makes this observation critical. The necessary volume increase is only about $5 \%$. You also need approximately a $5 \%-15 \%$ increase in the length of your incision. When you have confirmed this to your satisfaction, you should then remove the device from the pocket and prepare to insert with the Aigis ${ }^{\mathrm{TM}}$ pouch.

The manufactures have recommendations on how the product is used. They consist of the following. First, it is recommended that the antibacterial envelope be completely immersed in whatever irrigation solution (sterile normal saline, with or without antibiotics) for a few seconds to moisten the product. Then, they suggest everting the envelope so that the seam line of the two halves is on the inside of the envelope rather than on the outside. Next, they suggest placing the device in the pouch such that the header is pointing in the direction that points the lead wires toward the opening of the pouch. Then, the device and leads are inserted into the pocket. The pouch is kept free of the incision.

Recognizing these suggestions, we generally modify the procedure in the following manner: we use only a portion of the envelop; in general, we cut the pouch and place the cut portion over the roof of the generator. We cut a flat plane that is roughly the size of the generator. With the nonabsorbable pouch, we chose to do this initially to prevent placing the foreign body on the bottom of the pocket, in the prepectoral fascia, with the thought in mind that if we needed to remove the envelop in the future, either as part of a pocket-ectomy 
or in order to free-up slack for leads at a generator change, dissecting it away from the petoralis muscle could be a very bloody process and could lead to injury of the leads. We chose to make it roughly the size of the generator to minimize the chance of fibril extrusion into the incision closure and to avoid the leads becoming incorporated into the capsule. After performing several change-outs or lead revisions that had used the nonabsorbable pouch in their initial surgery, we found that this was probably unnecessary. The pouch becomes incorporated into the capsule that forms around all devices without changing the characteristics of the capsule to any significant degree. When performing a change out, if you are not aware that a nonabsorbable pouch was utilized, it is possible you will not even notice its presence. If you are paying careful attention, however, you will notice the now-clear (rather than bright yellow) strands in the dissected capsule. This is a very important point if you are removing an infected device, which will be addressed in more detail later.

Whether you use the whole or only a portion of the pouch, it is imperative that when you close your incision you are careful that you do not catch the filaments of the pouch with your needle. If you have chosen to cut the pouch, per below, it is even more important that you do not have a rough edge of the nonabsorbable product directly under your suture line as there is a risk of a fibril extruding through the suture line that can become a chronic foreign body.

When we use the absorbable pouch, we still choose to use only a flat plane, shaped like the generator. However, instead of placing it over the generator, we place it under the generator. We have three reasons for this. First, we want to get the antibiotics closer to the leads and can, which we perceive as the portion at a higher risk of surgical site contamination leading to infection due to bacterial adherence characteristic of polyurethane and silicone. Second, we want to get the bioabsorbable polymer into the deep tissue to maximize its exposure to bioenzymatic processes. Surgical literature suggests that those dissolvable suture rates of dissolution are dependent on sufficient environmental exposure to a wet environment. Thirdly, we want to minimize any chance of cut edges being inadvertently drawn into the incisional closure.

\section{How do our modifications (which are off-label use!), alter efficacy of the pouch?}

The areas we see as potential concern relate the following: decrease in antibiotic concentration, change in morphology, and change in device surface contact with the envelop.

We hypothesize that modifications do not significantly decrease the efficacy of the antibiotic due to the amount that is present in the envelop (antimicrobial agents in amounts of $8.0 \mathrm{mg}$ rifampin and $5.1 \mathrm{mg}$ minocycline [medium size], and $11.9 \mathrm{mg}$ rifampin and $7.6 \mathrm{mg}$ minocycline [large size]). These doses are over $100 \times$ the oral doses recommended as treatment; thus, we feel that utilizing only $40 \%$ of the surface area of the envelop still contains a vast safety margin of drug concentration.

As far as morphology change is concerned, having a rough cut edge instead of the smooth seam is definitely an increase in risk of the fibrils extruding into the wound closure and a careful observation to closure technique is necessary.

The other issue is surface area contact. The manufacturers hypothesized that having $100 \%$ of the generator encased in the pouch and thereby in contact with the antibiotics would prevent the biofilm adhesions that staph and strep are known to form on device surfaces - refer back to biofilm discussion under organisms. The theory was that the contact would be a factor in preventing biofilm adhesion to the device. However, we believe the overkill factor of the antibiotic concentration vastly outweighs the necessity of making $100 \%$ contact with the device surface. In order for a biofilm to develop, there would need to be a sufficiently high number of organisms present. We believe the antibiotic volume in the wound obviates the needs for complete surface contact.

\section{Generator exchange/removal of a pulse generator form an incorporated envelope}

When performing device exchange, you may treat the Aigis ${ }^{\mathrm{TM}}$ envelop the same as you would any chronic capsule. You may choose to cut into the capsule and carefully extract the generator or you may choose to perform a complete capsulectomy. Either way, you will need to open the capsule. If you decide to perform a capsulectomy, consider carefully where your incision will be in order to give yourself the best access to the whole capsule, as tunneling laterally to reach a capsule is more difficult than choosing to cut down right in the center of the capsule.

First, surgically expose the envelope, whether laterally via tunneling or cutting directly down over the center. Make an incision superior to the device, approximately the width of the generator. The most difficult aspect is taking care not to damage the leads, which may be wending in and out of the capsule. Compared to a capsule without the Aigis ${ }^{\mathrm{TM}}$ incorporated into it, the largest difference we have observed is the means by which the leads egress the capsule. They may be almost entirely inside, if they were wrapped around/ under the device and placed in the pouch, or may be almost entirely outside, if they were placed so that they extruded out 
of the opening of the envelope. As per usual in a generator change out, extreme care must be taken to free-up sufficient slack on the leads to be able to disconnect the electrode leads and reconnect them without being under too much tension. We recommend blunt dissection with a hemostat as much as possible. The Aigis ${ }^{\mathrm{TM}}$ does increase the tensile strength of the capsule in most cases and may require careful use of electrocautery to free up the leads. This puts the leads at increased risk of lead trauma. A plasma knife may be an excellent choice in this situation. Remove the device through the opening. A new generator may be inserted into the envelope through the side opening. Connect the electrical leads. A new Aigis ${ }^{\mathrm{TM}}$ pouch can be utilized to make a "pouch sandwich" if you choose; the absorbable may be preferable for a change out that has had a previous envelope in place. Suture the envelope/capsule should be closed per your standard practice and complete the procedure following your standard surgical protocol.

Of note, we have yet to see a heavily calcified capsule during a device change out in the presence of a previously utilized Aigis ${ }^{\mathrm{TM}}$ and wonder if this correlates with a lack of subclinical infections. This is entirely supposition based on personal observation with comparatively low numbers.

In the Atlanta Veterans Affairs Medical Center system, the Aigis ${ }^{\mathrm{TM}}$ envelope comes through our prosthetics department. We are permitted to have a certain number on hand and must report the statistics of our used implants to obtain replacements. The company provides a traceability label, which identifies the type, size, and lot number of the prosthesis. This is attached to the foil label in every package. In addition, we recommend that the data be entered into the device by the device representative, nurse or technician, who is interrogating and programming the implant so that it is in the permanent record. That alerts another device-implanting physician who may not have access to old medical records to the presence of the Aigis ${ }^{\mathrm{TM}}$ for our mobile patients.

\section{Pros and cons of adsorbable versus nonadsorbable}

After our initial few months of experimentation with the Aigis ${ }^{\mathrm{TM}}$ envelope, we felt strongly that the pros outweighed any cons and started using it in nearly $100 \%$ of our patients. There were only two subsets of patients in whom we were reluctant to implant: subpectoral device pockets and patients with extreme cachexia. In the first case, we were concerned about access and bleeding should the Aigis ${ }^{\mathrm{TM}}$ need to be removed from the subpectoralis space. In the second case, we were concerned about any excessive volume in a patient with extremely thin skin; however, this clearly needs to be addressed on a case-by-case basis as these patients are also likely at a higher risk for a system infection. While we never put the nonabsorbable envelop in a patient into the subpectoralis space, we have placed it on top of the prepectoral fascia after closing the muscle layer (an off-label use). Moreover, once we began using the absorbable envelop, we did so in all subpectoral implants.

\section{Case report}

The following photograph comes from a 55-year-old man with a medical history significant for end-stage renal disease who was implanted with a dual-chamber pacemaker for sick sinus syndrome at another institution. A nonresorbable Aigis $^{\mathrm{TM}}$ envelop was utilized during his surgery. The patient developed a wound infection 10 weeks after implant, and the patient was referred to a cardiothoracic surgical service at a different hospital to have his device extraction performed. The extracting physician was not aware that an Aigis ${ }^{\text {TM }}$ pouch was inserted at the initial implant and failed to remove it at explant. The patient presented to our hospital approximately 2 months later with the Aigis ${ }^{\mathrm{TM}}$ envelop protruding through his old incision (Figure 1). This highlights the importance of good documentation of the use of the Aigis ${ }^{\text {TM }}$ envelop. If

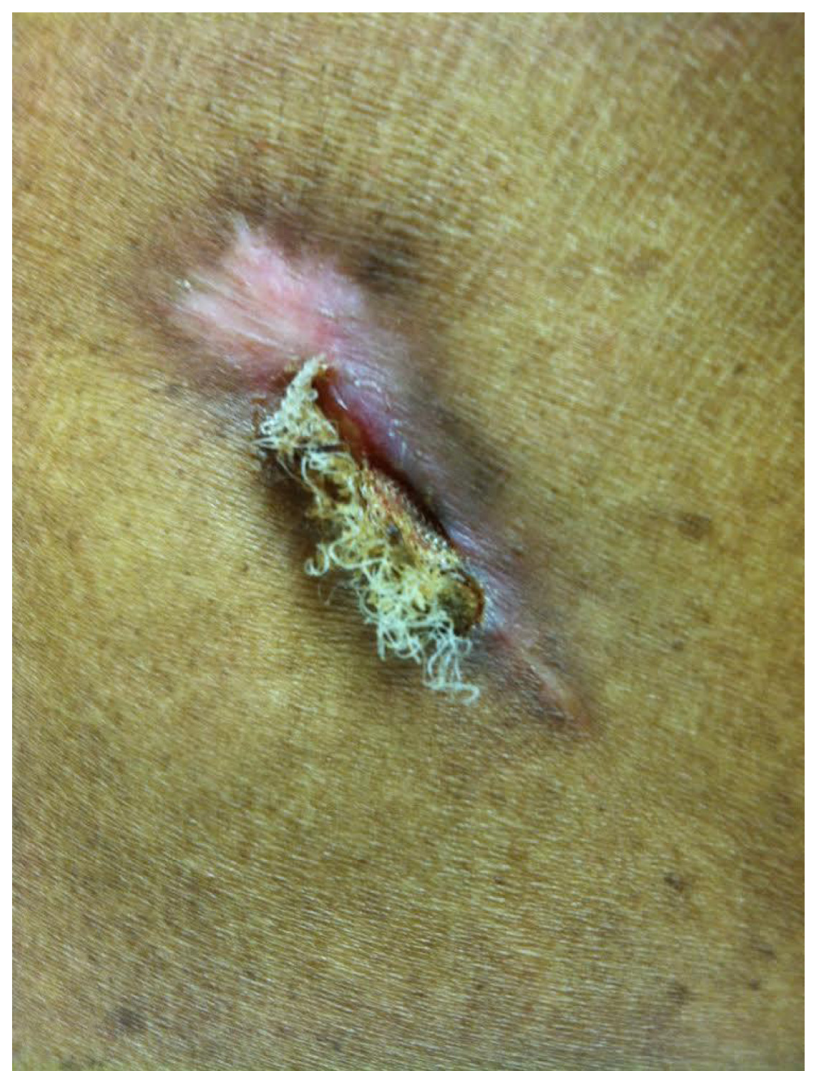

Figure I Explanted device with Aigis ${ }^{\mathrm{TM}}$ envelop abandoned, dehiscence. 
device extraction is performed, it is important to remove the Aigis $^{\mathrm{TM}}$ completely as the risk of future nonhealing wound, or wound dehiscence, is anticipated to be high in the care of a retained foreign body.

\section{Conclusion}

CIED is associated with significant morbidity, mortality, and cost. Despite meticulous adherence to anti-infective protocols when performing device surgery, infections continue to increase as we implant more patients, who are older and often have many comorbidities. The FDA-approved Aigis ${ }^{\text {TM }}$ antimicrobial pouch is a fairly new implantable pouch that provides additional protection against infection. Although most of the clinical research data to date is retrospective, the two prospective trials, Centurion and Citadel show very positive interim results. We all eagerly await the final results of those trials which are anticipated to be presented during 2015.

\section{Disclosure}

The authors report no conflicts of interest in this work.

\section{References}

1. Greenspon AJ, Patel JD, Lau E, et al. 16-year trends in the infection burden for pacemakers and implantable cardioverter-defibrillators in the United States 1993 to 2008. J Am Coll Cardiol. 2011;58:1001-1006.

2. Baddour LM, Bettmann MA, Bolger AF, et al. Nonvalvular cardiovascular device-related infections. Circulation. 2003;108:2015-2031.

3. Baddour LM, Epstein AE, Erickson CC. Update on cardiovascular implantable electronic device infections and their management. Circulation. 2010;121:458-477.

4. Byrd CL, Wilkoff BL, Love CJ, Sellers TD, Reiser C. Clinical study of the laser sheath for lead extraction: the total experience in the United States. Pacing Clin Electrophysiol. 2002;25:804-808.

5. Ferguson TB Jr, Ferguson CL, Crites K, Crimmins-Reda P. The additional hospital costs generated in the management of complications of pacemaker and defibrillator implantations. JThorac Cardiovasc Surg. 1996;111:742-775.

6. Sohail MR, Uslan DZ, Khan AH, et al. Management and outcome of permanent pacemaker and implantable cardioverter-defibrillator infections. J Am Coll Cardiol. 2007;49(18):1851-1859.
7. National Nosocomial Infections Surveillance (NNIS) System Report. National nosocomial infections surveillance (NNIS) system report, data summary from January 1992 through June 2004, issued October 2004. Am J Infect Control. 2004;32(8):470-485.

8. Sohail MR, Uslan DZ, Khan AH, et al. Infective endocarditis complicating permanent pacemaker and implantable cardioverter-defibrillator infection. Mayo Clin Proc. 2008;81(1):46-53.

9. Rohacek M, Weisser M, Kobza R, et al. Bacterial colonization and infection of electrophysiological cardiac devices detected with sonication and swab culture. Circulation. 2010;121(15):1691-1697.

10. Kolodzinska A, Kutarski A, Kozlowska M, et al. Biodegradation of the outer silicone insulation of endocardial leads. Circ Arrhythm Electrophysiol. 2013;6:279-286.

11. Hansen LK, Berg K, Johnson D, Sanders M, Citron M. Efficacy of local rifampin/minocycline delivery (AIGIS $(\mathrm{RX})^{\circledR}$ ) to eliminate biofilm formation on implanted pacing devices in a rabbit model. Int J Artif Organs. 2010;33(9):627-635.

12. Agostinho A, James G, Wazni O, Citron M, Wilkoff BD. Inhibition of Staphylococcus aureus biofilms by a novel antibacterial envelope for use with implantable cardiac devices. Clin Transl Sci. 2009;2(3):193-198.

13. Bloom HL, Constantin L, Dan D, et al; COoperative Multicenter study Monitoring a CIED ANtimicrobial Device Investigators. Implantation success and infection in cardiovascular implantable electronic device procedures utilizing an antibacterial envelope. Pacing Clin Electrophysiol. 2011;34(2):133-142.

14. Kolek JM, Dresden WF, Wells QS, Ellis CR. Use of an antibacterial envelope is associated with reduced cardiac implantable electronic device infections in high-risk patients. Pacing Clin Electrophysiol. 2013;36(3):354-361.

15. Mittal S, Shaw RE, Michel K, et al. Cardiac implantable electronic device infections: incidence, risk factors, and the effect of the AigisRx antibacterial envelope. Heart Rhythm. 2014;11(4):595-601.

16. Gould PA, Krahn AD. Complications associated with implantable cardioverter-defibrillator replacement in response to device advisories. JAMA. 2006;295(16):1907-1911.

17. Krahn AD, Lee DS, Birnie D, et al; Ontario ICD Database Investigators. Predictors of short-term complications after implantable cardioverterdefibrillator replacement: results from the Ontario ICD database. Circ Arrhythm Electrophysiol. 2011;4(2):136-142.

18. The Heart Rhythm Society's. MDT in mile high $4 \mathrm{~F}$ citadel/centurion study interim analysis: use of an antibacterial envelope is associated with very low 90-day CIED infection rates heart rhythm 2013, 34th annual scientific sessions. presented in: late-breaking clinical trials session III; Saturday, 8:00 am - 9:30 am; May 11, 2013; Denver.

19. Henrikson CA. Citadel \& Centurion studies. Poster presented at European Heart Rhythm Association (EHRA). Cardiostim. 2014.
Medical Devices: Evidence and Research

\section{Publish your work in this journal}

Medical Devices: Evidence and Research is an international, peerreviewed, open access journal that focuses on the evidence, technology, research, and expert opinion supporting the use and application of medical devices in the diagnosis, treatment and management of clinical conditions and physiological processes. The identification of novel

\section{Dovepress}

devices and optimal use of existing devices which will lead to improved clinical outcomes and more effective patient management and safety is a key feature. The manuscript management system is completely online and includes a quick and fair peer-review system. Visit http://www. dovepress.com/testimonials.php to read real quotes from authors. 